\title{
Experimental Investigations in Particle Physics at Intermediate Energies
}

\section{Performance Report}

for January 1st, 1990 to November 30th, 1990

\author{
L.B. Auerbach, V.L. Highland, C.J. Martoif \\ K.W. McFarlane, C. Guss and S. Kettell \\ Physics Department, Temple University \\ Philadelphia, PA 19122
}

July 24,1990

\section{DISCLAIMER}

This report was prepared as an account of work sponsored by an agency of the United States Government. Neither the United States Government nor any agency thereof, nor any of their employees, makes any warranty, express or implied, or assumes any legal liability or responsibility for the accuracy, completeness, or usefulness of any information, apparatus, product, or process disclosed, or represents that its use would not infringe privately owned rights. Reference herein to any sperific commercial product, process, or service by trade name, trademark, manufacturer, or otherwise does not necessarily constitute or imply its endorsement, recommendation, or favoring by the United States Government or any agency thereof. The views and opinions of authors expressed herein do not necessarily state or reflect those of the United States Government or any agency thereof.

\section{Prepared for}

The U.S. Department of Energy

(Division of High Energy and Nuclear Physics) Under Contract No. DE.FG02-88ER-40389 
Statement of unobligated balances for Contract No. DE-FG02-88ER-40389 (Division of High Energy and Nuclear Physics) The U.S. Department of Energy with Temple University K.W. McFarlane, Principal Investigator

We expect no unobligated balance at the end of the current period. 


\section{Disclaimer}

This report was prepared as an account of work sponsored by the United States Government. Neither the United States nor the United States Department of Energy, nor any of their employees, nor any of their contractors, subcontractors, or their employees, makes any warranty, express or implied, or assumes any legal liability or responsibility for the accuracy, completeness or usefulness of any information, apparatus, product, or process disclosed, or represents that its use would not infringe privately owned rights. 


\begin{abstract}
The major empriasis of this project continues to be on fundamental symmetries and parameters of the Standard Model. The primary project in the current period has been BNL E791, a search for the decay $K_{L}^{0} \rightarrow \mu e$, which would violate the rule of separate lepton number conservation. The technique uses a drift-chamber spectrometer, with particle identification, picking out candidate decays from 2-particle triggers by measuring the two-particle invariant mass $m$, and the collinearity angle $\Theta_{c}$. For reconstructed $K_{L}^{0} \rightarrow \pi^{+} \pi^{-}$decays the rms resolution in $m$ was $\sim 1.4$ $\mathrm{MeV} / \mathrm{c}^{2}$, and in $\Theta_{c}$ it was $\sim 0.3 \mathrm{mrad}$; these values match the design specifications. The data from the 1988 run of E971 have been published.

The 1989 run was completed in May 1989, and all the 2,900 data tapes were analysed using two computing centers: the BNI, center and the Cornell Supercomputer facility. A similar amount of data, from the run completed in May 1990, will also be analyzed in the current period. A preliminary analysis of the 1989 data give an upper limit for the branching fraction for $K_{L}^{0} \rightarrow \mu e$ of $(8.4 \pm 0.1 \pm 0.4) \times 10^{-11}(90 \%$ C.L.) and a limit of $(11.4 \pm 0.1 \pm 0.6) \times 10^{-11}(90 \%$ C.L. $)$ for $K_{L}^{0} \rightarrow e e$. We have a new (preliminary) value of the branching fraction for $K_{L}^{0} \rightarrow \mu \mu$ of $(7.6 \pm 0.5 \pm 0.4) \times 10^{-9}$, with a sample of 314 events. The limit on the decay $K_{L}^{0} \rightarrow \mu e$ places a lower limit on the mass of a new particle mediating such decays of $72 \mathrm{TeV}$. These are the best limits on separate-lepton-number-violating processes in $K_{L}^{0}$ decay.

We will combine the results for the two runs to achieve a sensitivity (for $K_{L}^{O} \rightarrow$ $\mu e)$ at the few $\times 10^{-11}$ level. We are confident that we can reject all backgrounds to $K_{L}^{0} \rightarrow \mu e$ at the $10^{-11}$ level.

The LSND (Large Scintillator Neutrino Detector), a search for neutrino oscillations at LAMPF, has been approved. The LCD project at LAMPF (measurement of $\nu-e$ elastic scattering) is in abeyance. Much of the technology developed for LCD will be applied on a smaller scalc to LSND.

Other neutrino work at Los Alamos, E764, will result in a final publication. This will include the best measurement of $\nu$-nuclear scattering, in $\nu_{\mu}{ }^{12} C$ inclusive cross sections. The measurement of the cross section for the exclusive reaction $\nu_{\mu}{ }^{12} C \rightarrow \mu^{-12} N$ is unique.

We have continued to participate in planning for experiments at CEBAF, and have joined the proposal to measure parity violation in $e, p$ scattering.

In a new development, Dr. Martoff is establishing a facility for fabrication of superconducting detectors of nuclear radiation; the equipment has been funded and is partly installed. Planned uses include searching for 'Dark Matter.'

In summary, the objectives for this year have been met.
\end{abstract}




\section{Contents}

Title page ........................ i

Disclaimer ..................... . . . . . . .

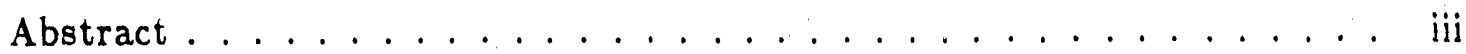

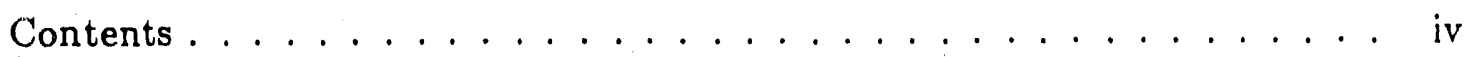

List of figures and tables ................... .

1. Introduction 1

2 Rare $K_{L}^{0}$ decays - BNL E791. 1

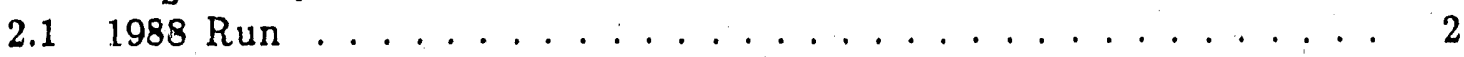

2.21989 and 1990 Runs . . . . . . . . . . . . . . 2

2.3 Level 1 logic . . . . . . . . . . . . . . . . . . . 3

2.4 Cherenkov Counter and Muon Hodoscope . . . . . . . . . . . . 4

2.5 General Comments .................... 5

2.6 Results . . . . . . . . . . . . . . . . . . 6

2.6.1 $K_{L}^{0} \rightarrow \mu e$ branching fraction . . . . . . . . . 8

2.6.2 $K_{L}^{0} \rightarrow \mu \mu$ and $K_{L}^{0} \rightarrow$ ee branching fractions . . . . . . . 9

2.7 Conclusions . . . . . . . . . . . . . . . . . 10

3 Neutrino interactions at LAMPF 14

3.1 Neutrino-electron scattering - LCD (E1015) . . . . . . . . . . . 14

3.2 Neutrino oscillations - LSND . . . . . . . . . . . . . . . 14

3.3 Neutrino interactions - E764 ............... 15

4 Parity Violation in polarized ep Scattering - CEBAF 15

5 Superconducting Detector Development 16

5.1 Dual Mode Si Detectors . . . . . . . . . . . . . . . . 17

5.2 Superconducting Quasiparticle Spectrometers . . . . . . . . 18

5.2.1 $\mathrm{Nb}-\mathrm{Al}_{2} \mathrm{O}_{3}-\mathrm{Nb}$ Tunnel Junctions as Radiation Detectors . . . . 18

5.2.2 Low Mass Ge Thermistors . . . . . . . . . . . . . . . . 18

5.2.3 Nb substrate characterization . . . . . . . . . . . . . . . 19

5.3 Setup of Microfabrication Laboratory . . . . . . . . . . . . . 19

6 Investigators' Time and budget $\quad 20$

7 Publications and Other References 21

7.1 Talks and Conference Reports . . . . . . . . . . . . . . . . 22

7.2 Other References . . . . . . . . . . . . . . . 23 


\section{List of Figures}

1 Histogram of invariant mass for $K_{L}^{0} \rightarrow \mu \mu$ events from ine 1989 daia. 7

2 Colinearity angle vs. invariant mass for $K_{L}^{0} \rightarrow \mu \mu$ events, 1989 data. 11

3 Colinearity angle vs. invariant mass for $K_{L}^{0} \rightarrow \mu e$ events, 1989 data. 12

4 Histogram of invariant mass for $K_{L}^{0} \rightarrow \mu \mu$ events from the 1990 data. 13

\section{List of Tables}

1. Mass bounds on new interactions, from rare processes . . . . . 8 


\section{Introduction}

We have continued to work on the structure of fundamental interactions, particularly on their basic symmetries and conservation laws. Our primary emphasis in the last year has been on the rare $K_{L}^{0}$ decay experiment at Brookhaven National Laboratory. The experiment is intended to look for lepton non-conservation, which is an assumption of the Standard Model without any theoretical basis. This work is in collaboration with six other institutions [9]. Good progress has been made on this work: two papers on the 1988 run have been published $[1,2]$, analysis of the 1989 data is almost complete and analysis of the 1990 data well underway. Some work has been done on a follow-on experimiment for the AGS "Booster Era."

A new proposal, for a 'Large Scintillator Neutrino Detector' (LSND - LAMPF E1173) $|12|$ to search for neutrino oscillations has been approved. The Large Cherenkov Detector (LCD) $|11|$ is currently in abeyance whil the collabration works on LSND. In the case of other neutrino work at LAMPF (E764), a final paper (on $\nu-C$ interactions) is in preparation. A related publication on the passage of electrons through aluminum $[3 \mid$ has been published.

A proposal to the DOE University Research Instrumentation program was funded and work has begun on this project [16]. The aim of the project is to fabricate nuclear radiation detectors of much higher sensitivity than existing types. The detectors will use superconducting tunnel junctions, and the technique is expected to have many applications, including the search for Dark Matter.

Details of the work on rare kaon decays are given in section 2 , of the work on LSND and LCD in section 3. Reference to work on planning for CEBAF is in section 4. The detector development effort led by Prof. Martoff is described in section 5. An outline of effort and funds expended is in section 6. Finally, publications resulting from the work are listed in section 7 .

\section{$2 \quad$ Rare $K_{L}^{0}$ decays - BNL E791}

The main goal of this project (E791) is to test the idea of separate lepton-number conservation to a new level of sensitivity. This is motivated by consideration of the puisibility that separate lepton number is not exactly conserved, as a result of attempts to develop more satisfactory theories than the Standard Model (SM). The branching fraction for $K_{L}^{0} \rightarrow \mu e$ is a critical test of such theories. Other goals proposed were a search for the decay $K_{L}^{0} \rightarrow e e$, the CP-violating decay $K_{L}^{0} \rightarrow$ 
$\pi^{\circ} e e$, a precise branching fraction for the decay $K_{L}^{0} \rightarrow \mu \mu$, and the CP-violating polarization in $K_{L}^{0} \rightarrow \mu \mu$.

Details of the physics goals $2 n d$ the design of the experiment are given in the original proposal [10]. In this section, we cutline our progress to date, and give current results.

In May 1987, we had a shakedown ruri (4 weeks); a first putlication resulted (15). For the 1988 run, we completed the remainder of the spectrometer, except for the ability to measure muon polarization. The data from the 1988 run have been analysed and published. $\{1,2\}$ The 1989 run began with studies of ways to improve the sensitivity (thicker target, smaller production angle, improved trigger electronics) and then settled into steady data-taking in a slightly improved mode. Some improvements were also made for the 1990 run. While the apparatus will be maintained until the analysis is complete, we do not expect to run again in 1991. Details are given in the following sections.

\section{$2.1 \quad 1988$ Run}

Results from the Spring 1988 run have been published in Physical Review Letters. One paper [1] gives upper limits for the branching fractions for $K_{L}^{0} \rightarrow \mu e$ and $K_{L}^{0} \rightarrow e e$ decays, while another $[2]$ gives a measurement of the branching fraction for $K_{L}^{0} \rightarrow \mu \mu$. The limit for $K_{L}^{0} \rightarrow \mu e$ is $2.2 \times 10^{-10}$ and for $K_{L}^{0} \rightarrow e e$ it is $3.1 \times 10^{-10}$, both at $90 \% \mathrm{C}$. L. The measured branching fraction for $K_{L}^{0} \rightarrow \mu \mu$ is $(5.8 \pm 0.6 \pm 0.4) \times 10^{-9}$, where the errors are statistical and systematic, respectively. This result is based on the observation of 87 events. Results from the 1989 run is described in a following section.

\subsection{9 and 1990 Runs}

In the 1988 data no candidate $K_{L}^{0} \rightarrow \mu e$ events survived reasonable cuts on track quality, particle identification, mass and collinearity. Even relaxing individual cuts introduced little background, so that it was reasonable to expect that a much larger data sample would still be background free, after reasonable cuts. Our efforts were therefore directed at taking more data in a reliable and efficient manner and at trying to increase the uaid rate by increasing the kaon flux. Reasonable improvements in particle identification were also sought.

For the 1990 run, the rectangular windows and beam stub at the end of the evacuated decay region were reylaced with a circular sailcloth/kapton wirdow and 
a new drift chamber was built to take advantage of the increased window area. Also the vacuum was improved from an average of 40 microns to 15 or less.

The data acquisition and on-line monitoring software were further improved for efficient long term operation. For these runs all eight $3081 / \mathrm{E}$ on-line computers were installed and operating. In this condition it was possible to take data with 4 to $5 \times 10^{12}$ protons/pulse on target and have virtually no dead time. At this rate it was not necessary to implement the level 2 trigger, which was found to have an inefficiency of $\sim 20 \%$. The beam rate was set by optimizing the rate of $K_{L}^{0}$ decay events that could be reconstructed from the drift chamber data. For the 1990 run, the on-line software was improveu in efficiency from $64 \%$ to above $85 \%$.

\subsection{Level 1 logic}

Temple is responsible for the L1 trigger for E791. Some modifications of the trigger logic were made, to improve deadtime and increase internal monitoring of the logic. At the beginning of the 1989 run a major effort was made to tighten the timing resolution of the 372 trigger counters in the level 0 trigger. An improvement from 20 ns to 14 ns was achieved.

Among the additions for 1990 the implementation of a new set of pulser triggers. These new triggers allow the simulation of $\mu \mathrm{e}, \mu \mu, \ldots$ triggers, by pulsing all relevant detector systems at the 'discriminator level'. This addition proved to be useful several times in diagnosing problems, as all electronics downstream of the discriminators can be tested. A second addition was the implementation of a 'semirandom' pedestal trigger. This trigger enables a sampling of the ADC pedestals uniformly throughout the $1.5 \mathrm{sec}$ beam spill and uniformly throughout the cycle of the $60 \mathrm{~Hz}$ line frequency. This eahancement should provide pedestal records that acurately reflect the offset of each ADC channel during our data collection period. In order to improve beam tuning operations we implemented a system whereby the L0 signal from our trigger scintillators was provided to the Main Control Room. This allowed for improved targetting, as feed back on targetting quality was available simultaneously with the ability to change all magnets. An auu ional improvement in data collection efficiency was implemented in the L 1 logic during the 1990 running period. A $6 \%$ increase in data rate was achieved by removing deadtime from the timing signal for the $\mathrm{L} 1$ trigger. This change in the $\mathrm{L} 1 \mathrm{timing}$ signal aiso removed an apparent inefficiency for latching the trigger bits decribing the origin of the trigger. A further addition to L1 was the ability to measure the 
live times of the separate trigger channels via the CAMAC scalers, enabling continuous monitoring of the relative live times of the scaled minimum bias triggers and the di-lepton triggers. A new Level 0 trigger was implemented to allow studying the drift chamber efficiency in the minimum bias trigger and for later studies with a beam plug in the downstream spectrometer magnet. Also two new trigger bits indicating the presence of a single muon on the left side or the right side of the spectrometer were added to aid various detector studies.

The Temple scintillation detector at the beam stop was maintained for use as an instantaneous beam intensity monitor. The live time of the experiment was measured on a day to day basis, where the live time is defined as the number of protons delivered while writing tape divided by the total number of protons delivered. At the start of the 1990 run the timing of all individual trigger hodoscope signals and muon hodoscope signals was adjusted. The timing of signals at all coincidences and for ADC gates and TDC starts and stops were rechecked and coincidence resolving times remeasured.

Studies of the performance of the LI system included measurements of the L1 trigger bit efficiencies, the deadtimes of scaled and unscaled triggers, the resolving times of the various coincidence circuits and the drift chamber efficiency in the L1 trigger. The efficiencies of the trigger bits after the change in the timing signal increased to essentially $100 \%$. Before the change the efficiencies of each of the trigger bits were measured to be $98.5 \%(\mathrm{MB}, \mu \mathrm{e}, \mathrm{e} \mu, \mu \mu)$ (The ee trigger bit efficiency was $98.0 \%$.) (The events lost due to lack of appropriate trigger bits were likely to be accidental events that would be rejected at a later stage.) The relative deadtimes of the scaled(i.e. MB) and unscaled(i.e. di-lepton) triggers follow the expected behaviour. These were studied by varying the length of the deadtime veto and by varying the scaling factor for the scaled triggers.

\subsection{Cherenkov Counter and Muon Hodoscope}

No major changes were made to the muon hodoscope and the Cherenkov counters, both Temple responsibilities. An inadvertent displacement of the Cherenkov counters was caused by the pressure of a Helium bag placed in the beam between the t.wo counters. About $25 \%$ of the data of the 1989 run were affected. The counter efficiency was reduced from $96 \%$ to $88 \%$ during those runs. The overall average efficiency is estimated to be $93 \%$. In November and December of 1989 , between the runs, the Cerenkov counters were removed from the beam-line, and the mirror 
alignments were checked, small adjustments beirg made where necessary. There were visual indications that there had been some slight deterioration in the mirror surfaces, so that some reduction in the efficiency of the counters was to be expected for the 1990 run.

The performance of the Cerenkov counters and the muon hodoscope during the 1990 run was monitored by using electron tracks from $\mathrm{K}_{L} \rightarrow \pi \mathrm{e} \nu_{e}$ events for the Ce renkov and muon tracks from $\mathrm{K}_{L} \rightarrow \pi \mu \nu_{\mu}$ events for the muon hodo scope. Our overall Cherenkov efficiency was $0.910 \pm 0.001$ and the muon hodoscope efficiency was $0.965 \pm 0.000$ over the entire 1990 run, and remained stable during the course of the run. The left detector efficiencies were in general agreement with the right side. The average number of photoelectrons collected by the hit Cerenkov phototubes for these electron tracks was about 3.0 to 3.2 . The average time distributions of these hits were also carefully monitored for any offset in the time distribution of the hits with respect to the trigger start for each counter during the run.

Time measurements in both systems were improved by installing new TDC's with least counts of 220 psec instead of the previous $2.5 \mathrm{nsec}$. The muon hodoscope was also moved a few inches upstream to place it nearly in contact with the iron absorber. Study of the previous data had suggested the possibility of a background caused by particles at wide angles that might hit the inner edge of the hodoscope without passing through the iron.

\subsection{General Comments}

In 1988 the angle of the secondary beam from the incident proton beam was $2.75^{\circ}$. It was hoped that the $K_{L}^{0}$ flux per proton could be increased by working at a smaller angle, since neutron interactions did not seem a limiting factor at $2.75^{\circ}$. A low intensity run at $1.875^{\circ}$ showed, however, that the increase in $K_{L}^{0}$ intensity was less than expected and was counterbalanced by a decrease in the event reconstruction efficiency, due to increased rates in the drift chambers. Some of the short-fall in intensity is due to reabsorption and scattering in the $\mathrm{Cu}$ target. For both 1989 and 1990 the angle remained at the original $2.75^{\circ}$.

The AGS continued to run at $24 \mathrm{GeV}$ primary energy in 1989 and 1990. This energy results in approximately $1 / 3$ reduction in kaon flux, relative the full energy of $29 \mathrm{GeV}$. This loss is partly compensated for by shorter cycle time, better machine reliability and a longer operating period due to lower power costs.

Data acquisition ceased in May 1990. Approximately 3,500 data tapes (of which 
2,900 were physics data tapes), each containing about 90,000 events, were accumulated in 1989 and about the same in 1990. We had expected more in the 1990 run, but radiation safety problems curtailed the AGS SEB run. All of the physics data tapes have been put through a first pass of analysis. This pass does track and vertex reconstruction. Events are retained if they have a satisfactory geometric reconstruction and then are consistent in reconstructed mass and angle with a twobody decay of the $K_{L}^{0}$ consistent with the identification of the decay particles by the muon hodoscope and Cherenkov counters. Acceptance criteria are quite loose at this stage. The rapid processing of the first pass was made possible by using the Cornell supercomputer in addition to the BNL 3090. The second pass is complete on the 1989 data, and will be completed on the 1990 data in the current period.

A major effort was made to re-measure the magnetic field just before the 1989 run. Improved magnetic shields had been installed on the first magnet (96D40) to reduce the field at the first drift chamber. In addition we believed that the experience gained from the first measurement would give us data of higher consistency and quality in a re-measurement. The large extent of the total magnetic field makcs a measurement at the $0.1 \%$ level extremely difficult with available appartus. The next stage of data analysis will rely on the magnetic field map which is being produced from the raw field measurements.

\subsection{Results}

The analysis of the 1989 data is nearing completion (the 1990 data will be added before publication). Preliminary results from the 1989 run are summarized here, and show that the experiment is proceeding as planned.

The final stage of the analysis is based on two parameters: collinearity and invariant mass. Two-track events are reconstructed and then selected on track quality and particle identification. The collinearity angle is the angle between the kaon direction (as found from the line joining the target to the reconstructed vertex) and the sum of the momentum of the two particles. The invariant mass is the invariant mass of the two particles. In the ideal case the collinearity angle is zero, and the invariant mass equal to the kaon mass.

In figure 1 we show the preliminary reconstructed mass spectrum for $K_{L}^{0} \rightarrow \mu \mu$ events, in figure 2 the collinearity angle vs. mass scatter plot for these events, and in figure 3 the scatter plot for $K_{L}^{0} \rightarrow \mu e$ events. There are about $300 K_{L}^{0} \rightarrow \mu \mu$ events in the 1989 sample, and we expect the same number in the 1990 sample; 
this will be the world's largest sample of such decays. Figure 4 shows the mass histogram for $K_{L}^{0} \rightarrow \mu \mu$ from a sample of the 1990 data. As can be seen, the rejection of background is excellent, so that our upper limits will be determired by the sensitivity.

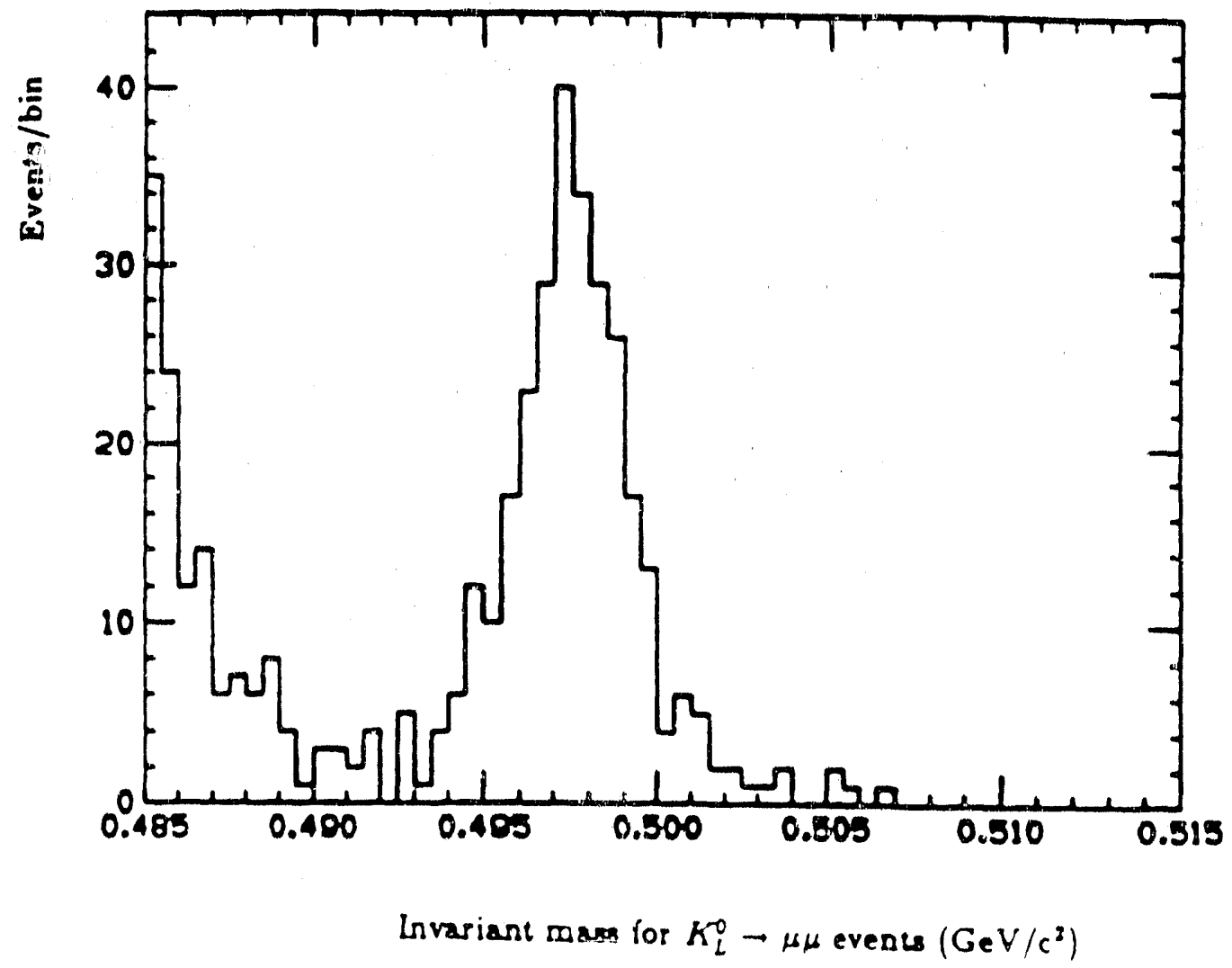

Figure 1: Histogram of invariant mass for $K_{L}^{0} \rightarrow \mu \mu$ events from the 1989 data, showing the excellent mass resolution. This is a projection of figure 2 , for the events with $\Theta_{c}$ less than $2 \times 10^{-6} \mathrm{rad}^{2}$.

Here we discuss some of the implications of the results from the 1989 run: the best limit on $K_{L}^{0} \rightarrow$ ee and on $K_{L}^{0} \rightarrow$ ee and the most precise measurement of the $K_{L}^{0} \rightarrow \mu \mu$ branching fraction. Since other experiments on $K_{L}^{0} \rightarrow \pi^{0} e e$, notably E731 at Fermilab and E845 at BNL. have much better sensitivity, we have stopped further work on this mode. As noted above, the analysis of the 1990 data is in progress; we expect about 500-600 $K_{L}^{0} \rightarrow \mu \mu$ events, and appropriate sensitivities for $K_{L}^{0} \rightarrow$ me and $K_{L}^{0} \rightarrow$ ee. 


\subsection{1 $K_{L}^{0} \rightarrow \mu e$ branching fraction}

The present repoitud values of $B\left(K_{L}^{0} \rightarrow \mu e\right)$, from the recent round of experiments, are:

BNL E791: $\leq(8.4 \pm 0.1 \pm 0.4) \times 10^{-11}(90 \% \mathrm{CL}), 1989$ data. $[1]$

BNL E780: $\leq 1.9 \times 10^{-9}(90 \% \mathrm{CL}),[17]$

$\mathrm{KEK}: \leq 4.3 \times 10^{-10}(90 \% \mathrm{CL}),[18]$

We expect the combination of our 1989 and 1990 data to give a limit of about half of our limit above.

We can compare $K_{L}^{0} \rightarrow \mu e$ to other rare processes in terms of sensitivity to new physics, showing that our $K_{L}^{0} \rightarrow \mu e$ search is probing physics at scales near 100 $\mathrm{TeV}$. We compare with two approaches: that of Cahn and Harari [19], referred to here as $\mathrm{C} \& \mathrm{H}$, who proposed a 'horizontal interaction' mediated by a new boson, and suppressed by its high mass; and with the 'effective Lagrangian' approach of Buchmuller and Wyler [20], which establishes a scale of a new interaction, rather than a particle mass. The results of the models of $C \& H$ and $B \& W$ are given in table 1 , which gives the lower limits using recent data $\mid 1,21,22,23,24]$.

\begin{tabular}{lccc}
\hline Process & $\begin{array}{c}\text { current } \\
\text { value }\end{array}$ & $\begin{array}{c}\mathrm{C \& H} \\
\text { bound } \\
(\mathrm{TeV})\end{array}$ & $\begin{array}{c}\text { B\&W } \\
\text { bound } \\
(\mathrm{Tev})\end{array}$ \\
\hline$K_{L}^{0} \rightarrow \mu e$ & $<8.4 \times 10^{-11}$ a & 70 & 150 \\
$K^{+} \rightarrow \pi^{+} \mu^{+} e^{-}$ & $<2.1 \times 10^{-10}$ & 40 & 43 \\
$\mu N \rightarrow e N$ & $<4.6 \times 10^{-12}$ & 190 & 340 \\
$\mu^{+} \rightarrow e^{+} e^{+} e^{-}$ & $<1 \times 10^{-12}$ & 80 & 186 \\
$\mu \rightarrow e \gamma$ & $<1.7 \times 10^{-11}$ & 20 & - \\
$\Delta m_{K}$ & $3.5 \times 10^{-6} \mathrm{eV}$ & 400 & 1000 \\
\hline
\end{tabular}

"This expt., 1989 data

"Conversion on $\mathrm{Ti} / \mathrm{Capture}$

Table 1: Comparison of mass bounds on new interactions obtained by simple dimensional arguments with bounds calculated following Cahn and Harari (C\&H) and Buchmuller and Wyler ( $B \& W)$. The bounds from $B \& W$ are bounds on the scale $\Lambda$ of a new interaction, rather than on the mass of a mediating particle. 


\subsection{2 $K_{L}^{0} \rightarrow \mu \mu$ and $K_{L}^{0} \rightarrow e e$ branching fractions}

The decay $K_{L}^{0} \rightarrow \mu \mu$ is the well-known CDA suppressed flavor-changing neutral current decay. The accepted value of $B\left(\bar{K}_{L}^{-} \rightarrow \mu \mu\right)$ (prior to BNL E791) was $\left(9.5_{-1.5}^{+2.4}\right) \times 10^{-9}[26]$. Recent values are:

BNL E791: $(7.6 \pm 0.5$ (stat) $\pm 0.4($ syst $)) \times 10^{-9}$, this expt., preliminary, 1989 data

BNL E791: $(5.8 \pm 0.6$ (stat) $\pm 0.4($ syst $)) \times 10^{-9}[2]$, this expt., 1988 data

KEK: $(8.4 \pm 1.1$ (stat. only, no syst. $) \times 10^{-9}[18]$

Theoretical investigations $[25]$ have been made of the contribution to the $K_{L}^{0} \rightarrow$ $\mu \mu$ rate by the intermediate process $K_{L}^{0} \rightarrow \gamma \gamma$. If this absorptive part of the amplitude for this decay were the only contribution, the branching ratio would be expected to be $B\left(K_{L}^{0} \rightarrow \mu \mu\right) \approx 1.20 \times 10^{-5} \times B\left(K_{L}^{0} \rightarrow \gamma \gamma\right)$. (This is often referred to as the 'unitarity limit.') After an experiment reported a value (later superseded by the measurements cited in ref. (26]) significantly below this, increased theoretical activity emphasized that other processes could decrease this branching ratio very little.

The calculations of the GIM mechanism, which suppress the decay in the quark model, have been extended to the six-quark Standard Model in several analyses. $\{28\}$ As a result, the deviation from rate predicted from the $K_{L}^{0} \rightarrow \gamma \gamma$ intermediate state can be used to constrain SM parameters in some models. For example, the model of Shrock and Voloshin gives (for large $m_{t}$ ) the 'structure-dependent' contribution to the branching fraction as $B_{S D} \approx 3.6 \times 10^{-11}\left(\mathrm{GeV} / \mathrm{c}^{2}\right)^{-4}\left(\operatorname{Re}\left(V_{t}^{*} V_{t d}\right)\right)^{2} m_{t}^{4}$.

The difference between our new result for $B\left(K_{L}^{0} \rightarrow \mu \mu\right)$ and the value predicted from the observed $K_{L}^{0} \rightarrow \gamma \gamma$ rate $\left(6.81 \pm 0.31 \times 10^{-9}\right)$ is $(0.8 \pm 0.8) \times 10^{-\theta}$. If $B_{S D} \leq 10^{-9}$ as implied by this result, and $m_{t} \sim 120 \mathrm{GeV} / \mathrm{c}^{2}$, this would place a limit of $\sim 5 \times 10^{-4}$ on $\operatorname{Re}\left(V_{t s}^{*} V_{t d}\right)$, compared with possible ranges up to $1.4 \times 10^{-3}$ in the SM, and greater if there are more than three families. Other models give smaller predictions, so that the constraint of $B\left(K_{L}^{0} \rightarrow \mu \mu\right)$ is less useful.

The prediction from $K_{L}^{0} \rightarrow \gamma \gamma$ for $B\left(K_{L}^{0} \rightarrow e e\right)$ is $2.5 \times 10^{-12}$. A measured rate significantly higher than this might indicate new physics. The present values of $B\left(K_{L}^{0} \rightarrow e e\right)$ are:

BNL E791: $\leq(11.4 \pm 0.1 \pm 0.6) \times 10^{-11}(90 \% \mathrm{CL})$. This expt., prelim. from 1989 data.

BNL E791: $\leq 3.1 \times 10^{-10}(90 \% \mathrm{CL}) \cdot[1\}$ 
BNL E780: $\leq 1.2 \times 10^{-9}(90 \% \mathrm{CL}) \cdot[17]$

$\mathrm{KEK}: \leq 5.6 \times 10^{-10}(90 \% \mathrm{CL}) \cdot[18]$

\subsection{Conclusions}

We have met the objectives for the current period: ro make the best measurement on $K_{L}^{0} \rightarrow \mu e$, improve limits on $K_{L}^{0} \rightarrow e e$ and make a more precise measurement of the $K_{L}^{0} \rightarrow \mu \mu$ branching fraction. We have published results from data taken in $1988[1,2]$. The data taken in 1990 will increase our sensistivity by a factor of two, and background is not yet a problem. 


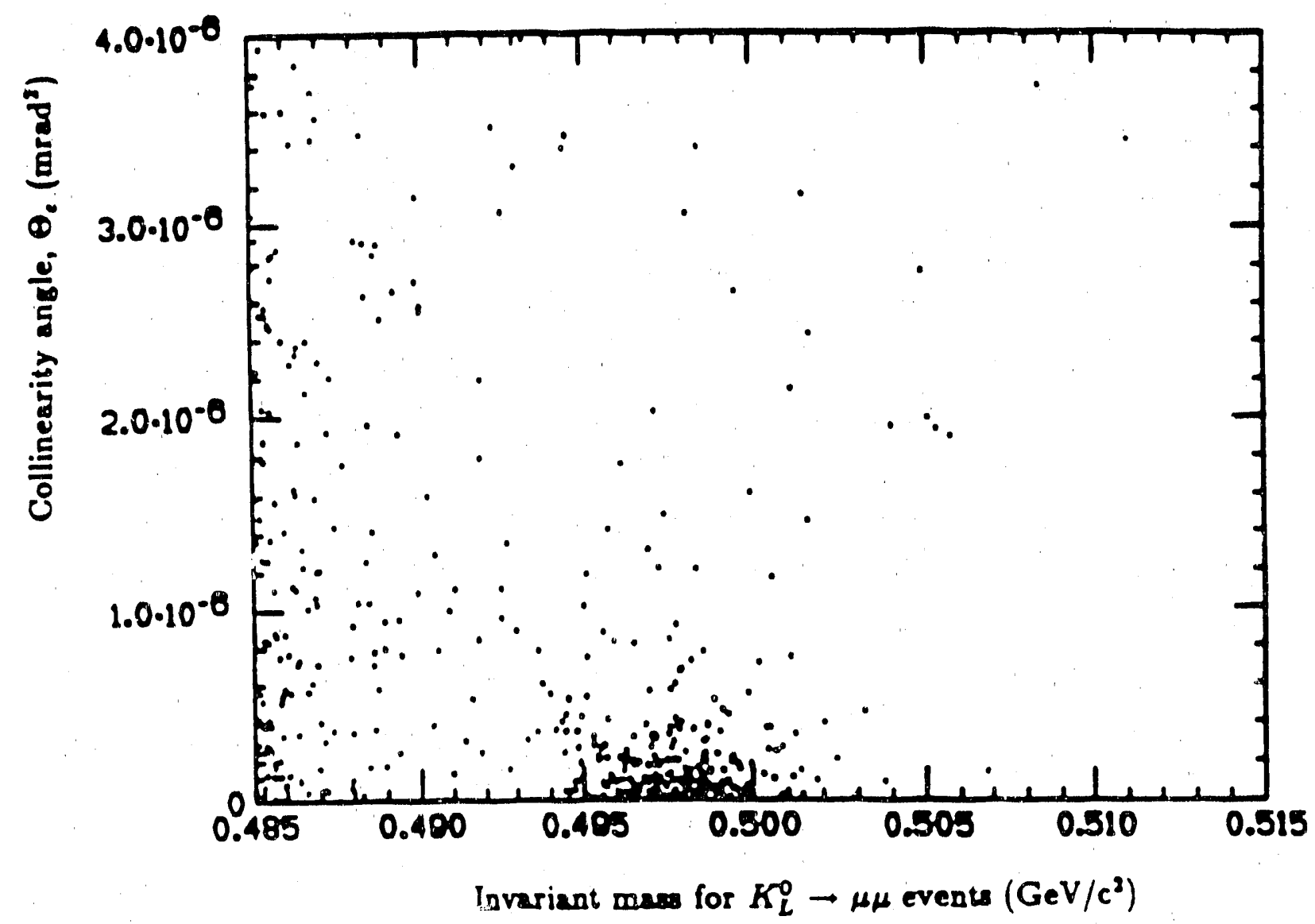

Figure 2: Plot of collinearity angle vs. invariant mass for $K_{L}^{0} \rightarrow \mu \mu$ events from the 1989 data, showing the exceilent mass and collinearity resolution. 


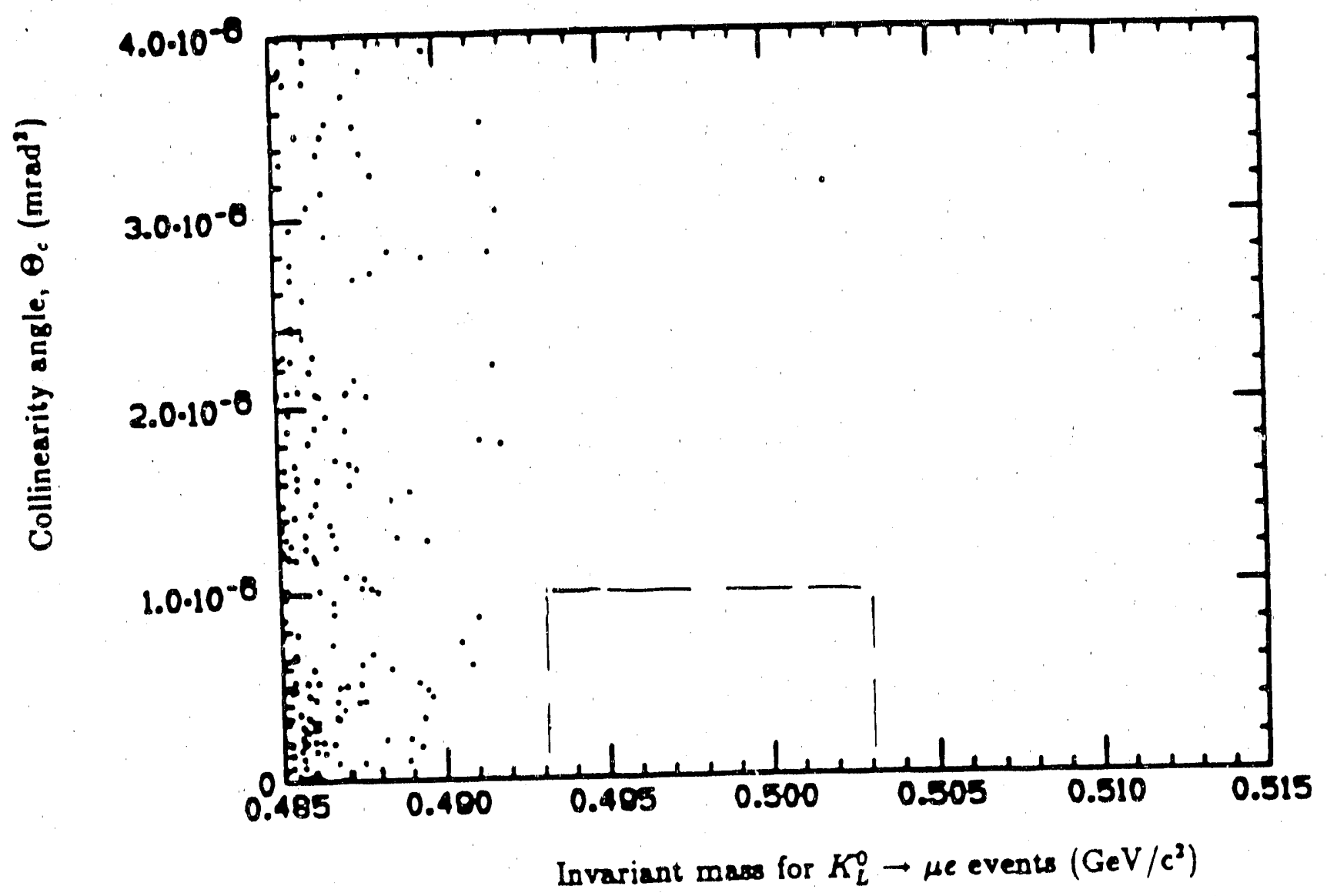

Figure 3: Plot of collinearity angle vs. invariant mass for $K_{L}^{0} \rightarrow \mu e$ events from the 1989 data, showing the low background in the signal region. 


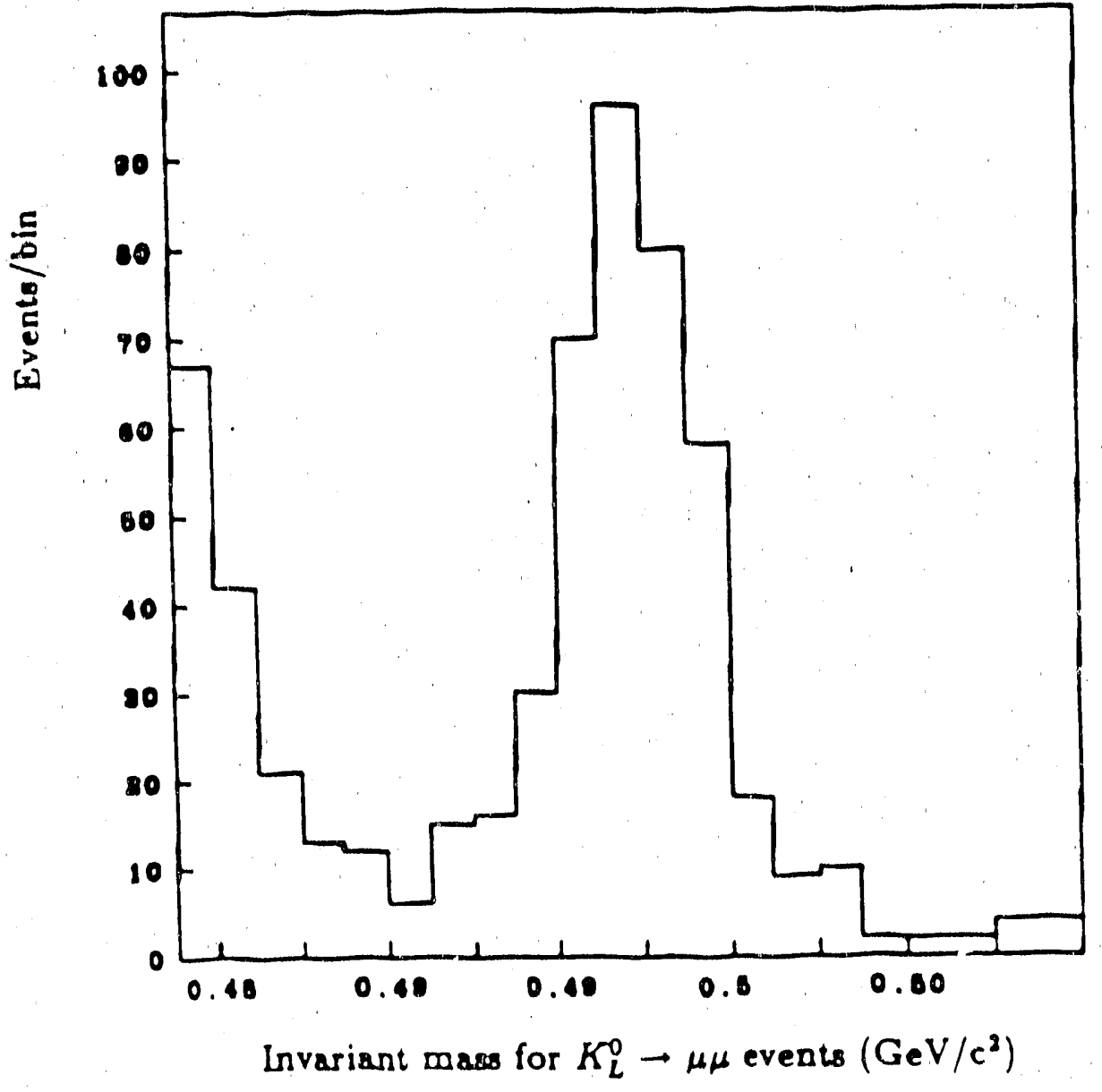

Figure 4: Histogram of invariant mass for $K_{L}^{0} \rightarrow \mu \mu$ events from the 1990 data. 


\section{Neutrino interactions at LAMPF}

\section{1 iveutrino-electron scattering - LCD (E1015)}

The LCD proposal [11] was to make a precise measurement of the neutrino-electron scattering cross-section at LAMPF. Using a beam-stop neutrino source and a waterČerenkov detector, the relative total crossections for elastic $\nu_{\mu} e, \nu_{e} e$, and $\nu_{\mu} e$ scattering will be measured with sufficient precision to fix $\sin ^{2} \theta_{W}$ to less than $1 \%$. This bears on the consistency of the parameters of the Standard Model, and is complementary to other measurements, of the $Z^{0}$ mass for example. The recent increase in the likely mass of the top quark $\left(m_{t}>77 \mathrm{GeV} / \mathrm{c}^{2}\right)$ has made LCD more important, by increasing the size of the $(\approx 7 \%)$ radiative corrections to which LCD is sensitive.

The project has passed through several phases. It was reviewed by a DOEappointed panel chaired by B. Barish, which made a favorable recommendation. Engineering studies indicate the feasibility of constructing the device at the site, and a financial review found that the estimated budget is reasonable. LCD spurred the development of new types of photomultipliers. Tests of a several examples of one manufacturer's tube (Burle Industries) have been carried out, and several other manufacturers have developed prototypes in response to our specifications. The U.S.-Canadian Sudbury Neutrino Observatory project is likely to adopt the Burle PMT that was developed for LCD; this tube will also be used for LSND.

The project has not yet been funded, and so we have, along with other memebers of the LCD collaboration, turned our attention to LSND.

\subsection{Neutrino oscillations - LSND}

This is a proposal $[12\}$ to search for neutrino oscillations using a smaller-scale prototype of LCD and an existing neutrino area at LAMPF that is equipped with a cosmic-ray veto shield. Its intent is to use LCD technology in a physics experiment, both as a development tool for the LCD project and to improve limits on neutrino oscillations by an order of magnitude over existing results. The LCD technology to be used consists of several elements: large PMT's immersed in a Cherenkov medium (with the addition of some scintillator but using both types of light), the data arquisition electronics, and similar reconstruction algcrithms. A detailed description is being submitted with our Continuation Application. This experiment will be a high-sensitivity search for neutrino oscillations in the appearance mode in the channels $\nu_{\mu} \rightarrow \nu_{e}, D_{\mu} \rightarrow D_{e}$; it will also yield measurements of the reactions 
$\nu_{e} C \rightarrow e^{-N}$ and $\nu C \rightarrow \nu C^{*}(15.11 \mathrm{MeV} \gamma)$. It will be a major improvement over Expts. 645 and 764 which were done previously at LAMPF. Temple will work with LANL on the question of what linuid scintillator (and in what quantity) will be added to the mineral oil which will constitute the primary liquid of the neutrino de cector. Tests have been done with cosmic rays and radioactive sources, and this summer more tests will be done in the test channel at LAMPF. Temple is building beam-counters for this work and will participate in the running of the tests and analysis of results.

\subsection{Neutrino interactions - E764}

Final calculations on the difficult question of the neutrino flux have been completed, allowing absolute values of $\nu$ cross sections to be given (with a $15 \%$ systematic uncertainty). These are the $\nu_{\mu} C$ inclusive scattering total cross section and the unique measurement of the exclusive reaction $\nu_{\mu}{ }^{12} C \rightarrow \mu^{-12} N$. The values will be compared with calculations based, for example, on the approach of Wal scka [29]. This comparison should result in better understanding of nuclei and their interaction with the well-understood $\nu$ probe.

Experimental work done on radiative processes occurring during the passage of electrons through a multi-layered Aluminum Scintillator sandwich has been published in Nuclear Instruments and Methods. $|3|$

The calculation of the neutrino flux in E764 has been completed, and a Physical Review paper is now in preparation that will give all of the results (oscillation of $\nu_{\mu}$ to $\nu_{e}, \nu_{\mu} \mathrm{C}$ cross-sections) of the experiment.

\section{Parity Violation in polarized ep Scattering - CEBAF}

Our primary interests in CEBAF are in the measurement of parity violation in electron scattering (from protons and helium), in the possibility of doing kaon physics using production of $\phi$ mesons as a source of kaons, and in measuring the neutral pion form factor. This work is in the study stage, with the parity work having reached the stage of a formal proposal.|13]

The proposal (CEBAF PR-89-023) was considered by the April 1990 meeting of the CEBAF Program Advisory Committee. While it was not approved, the PAC did feel that it was a valuable area in which CEBAF could contribute, and that 
the Laboratory not do anything that qwould preclude delivering polarized electron beams with the needed precision.

At Temple we will design and construct (in the current period) some prototype scintillation counters for the detection of recoil protons from a liquid hydrogen target with a 200 microamp beam of electrons incident on it. To withstand the high flux of low energy photons and electrons (the "gamma flash"), careful design is necessary. First of all the scintillator should probably be made very thin to take advantage of the high ionization of the protons while minimizing photon and neutron interactions. For about the same cost one could have either single pieces of scintillator with two phototubes for meantiming or two pieces with single tubes in opposed directions.

We will experiment with gating the dynode or grid electrodes of the photomultipliers. If the gating can be done fast enough we may be able to minimze the effect of the gamma flash on the phototubes. Tubes do not immediately recover from being gated off; this may introduce and undesirable source of variation in detection efficieny. If the effect of gating on tube gain were rate dependent it would add a potential source of spurious correlation with the beam polarization. In that case the techniciue could not be used.

With these measures, we are optimistic that suitable proton recoil counters can be made to work in the CEBAF environment. After bench studies of prototype counters, we would plan to take several sets of counters to the Bates electron accelerator and test them in as realistic a fashion as possible. L. Cardman believes he could pulse his electron source in a way that would simulate a chopped CEBAF beam structure at Bates.

\section{Superconducting Detector Development}

This part of the project is an effort to develop extremely sensitive detectors of nuclear radiation, based on a variety of low-temperature technologies. A combined DOE-Temple equipment fund of $\$ 450,000$ is being used to set up a device fabrication and test facility. The funds were provided under a DOE University Research Instrumentation (URI) grant of $\$ 250,000$. These devices can be sensitive to low energy nuclear recoils, as from neutrino or 'Dark Matter' scattering; they can be fast (in the nanosecond range); they can measure position with micron precision and they can be radiation hard. Thus there are many possible applications.

The following sections detail progress in setting up this facility. Five Temple 
students (Zhang, Garzarella, Fuller, Wang and Works) have been involved in this, meeting one of the goals of the DOE URI program: on-ca us involvement of students in nuclear physics programs.

\subsection{Dual Mode Si Detectors}

These detectors would measure both ionization and phonon production by incident radiation. This would permit rejection of e.g. ${ }^{3} \mathrm{H}$ decays in a Si dark matter detector, since a low ionization-to-phonon ratio is a sensitive indicator of high LET radiation.

1. An experiment was carried out at Ohio University in collaboration with Ohio $U$ and Stanford scientists, to measure the ionization-to-total energy deposition ratio for $\mathrm{Si}$ recoils in $\mathrm{Si}$ at $4-64 \mathrm{KeV}$. Neutron elastic scattering was used, with pulse shape discrimination and detection of scattered neutrons in coincidence with the Si pulses. This work has been published.|5]

2. Collaboration with B. Neuhauser of S.F.S.U. and F.P. Ziemba on dual mode silicon detectors was initiated. A first test was carried out at SFSU with Temple nuclear spectroscopy electronics and personnel. A high purity Si diode with degenerately doped (diffused junction) contacts was shown to detect alpha particles with the same efficiency and pulse height at $4.2 \mathrm{~K}$ as at room temperature. Additional devices are being fabricated at SFSU by Temple graduate student $Y$. Zhang.

3. Dewar station and probe fabricated from scratch at Temple for detector tests.

4. Further tests with improved diffused junction detectors showed better performance (faster risetime, lower noise level) at temperatures from 1.3 to $4.2 \mathrm{~K}$ than at room temperature. This work has been submitted for publication in Nuclear Instruments and Methods.

5. Tests of ion implanted uevices (thinner contacts for less attenuation of phonons) have revealed unsatisfactory performance below $70 \mathrm{~K}$.

6. Diffused junction devices will be equipped with $\mathrm{Al}$ and $\mathrm{Ti}$ superconducting transition edge phonon transducers (SCTD's) for dual mode detector tests. These tests will be complete by September. 


\subsection{Superconducting Quasiparticle Spectrometers}

This is a complex project which has been divided into several elements, with a student assigned to each.

\subsection{1 $\mathrm{Nb}-\mathrm{Al}_{2} \mathrm{O}_{3}-\mathrm{Nb}$ Tounel Junctions as Radiation Detectors}

1. A collaboration with TRW Superconducting electronics group was initiated. A test chip with 40 trilayer junctiona on Si substrate was fabricated at TRW from a mask set made by Temple.

2. Two dedicated probes for trilayer junction tests were fabricated at Temple; one designed by Ed Kaczanowicz for temperatures above $1.3 \mathrm{~K}$, and another designed by $\mathrm{B}$. Neuhauser which maintains temperatures as low as $0.265 \mathrm{~K}$ for as long as 24 hours. Final assembly and initial low temperature tesi of the ultra-low temperature probe were carried out at Stanford by SFSU, Temple, and Stanford University collaborators.

3. Ultrasonic wire bonding techniques were developed at Temple to permit connection of trilayer chip to room temperature electronics.

4. Final assembly and mounting of the trilayer test chip in the $1.3 \mathrm{~K}$ probe is now underway. Initial tests should be complete by $15 \mathrm{July}$.

5. Ultra-low noise amplifier designs using SONY GaAs-FET front end at $4.2 \mathrm{~K}$ are under development for later stages of trilayer device tests.

\subsubsection{Low Mass Ge Thermistors}

These devices are a possible alternate transducer for use with quasiparticle spectrometers.

1. Detailed calculations were carried out to model the quasiparticle transport and thermodynamic properties of quasiparticle spectrometers with thin film trapping layers. The trapping layer temperature is monitored by a low mass thin film Ge thermistor. The calculations indicate that thin film Ge thermistors having properties easily attainable with simple fabrication techniques should perform adequately as radiation detectors (roughly ten per cent resolution at $1 \mathrm{KeV}$ energy deposit). 
2. Expertise in various techniques for thin film deposition was developed at Temple. Ag, Au, Al films with overlayers and underlayers of $\mathrm{Ge}, \mathrm{Au}-\mathrm{Ge}$, and $\mathrm{Sb}$ Ge were deposited. A disused Sloane Angstrometer was put into service and thickness measurements were checked against gravimetry. Superconducting Al films were deposited and tested at low temperature.

3. Literature techniques for Ge and Au-Ge thin film thermistor deposition were researched and expertise is being developed at Temple. Good quality pure Ge films were successfully prepared by high vacuum evaporation. Au-Ge and Sb-Ge film devices with Au electrode structures have been prepared which are expected to be suitable for low temperature thermometry. Final mounting in an existing 1.3 ris probe, and connection to room temperature electronics, is in progress. Low temperature tests of these initial devices should be underway by 30 June.

\subsubsection{Nb substrate characterization}

1. High purity ("RRR grade") $\mathrm{Nb}$ stock $1 \mathrm{~mm}$ thick was purchased and its residual resistivity ratio (RRR) checked between room temperature and 4.2 $\mathrm{K}$. The RRR was only 250 , but this was judged sufficient for initial tests with such thin absorbers.

2. Metallographic polishing techniques are being developed at Temple to prepare $\mathrm{Nb}$ with adequate surface finish for microfabrication of transducers. Mechanical polishing and electropolishing are being researched, with guidance from electron micrographs obtained in the biology department's facility. Samples have also been sent out for commercial polishing.

3. Test devices with $\mathrm{Nb}$ absorbers, Al trapping layer3, and macroscopic Ge based thermistors, will be fabricated by $30 \mathrm{July}$. More sophisticated devices with trilayer junctions possibly SCTD's should be fabricated by September.

\subsection{Setup of Microfabrication Laboratory}

This is a complex project involving a large number of technical and adminstrative/bureaucratic issues.

1. Ed Kacazanowicz has performed airflow and air conditioning calculations to estimate the cleanroom equipment parameters (number of HEPA modules, 
air conditioning capacity, etc.)

2. A large number of cleanroom contractors have been interviewed and have visited the Temple site. Several have prepared preliminary project descriptions and cost estimates for a Class 10,000 cleanroom installation in the available room. These were compared to each other and to our own calculations.

3. A formal bid document project description has been prepared and submitted to the Office of the Vice Provost for Research and Program Development. This office will oversee the preparation of the full bid document. This document was promised for 1 June, but is still in preparation.

4. Two large new HEPA filter banks providing approximately half the capacity required for the cleanroom were bought in the secondary market. These will provide a savings of roughly $\$ 15,000$ in the project construction.

5. Nuclear spectroscopy electronics and an $\mathrm{AC}$ resistance bridge/ temperature controller were bought new.

6. Several necessary equipment items have been bought used, including a reactive ion etcher and an ultrasonic wire bonder.

7. A sputtering system (three gun, sputter-up high vacuum $\mathrm{DC} / \mathrm{RF}$ system with load-lock) for trilayer deposition has been designed and ordered from Kurt J. Lesker Co. Approval drawings have been reviewed and approved. The system will be delivered about 15 July.

8. Ed Kaczanowicz has retrofitted an existing vacuum evaporation with watercooled DC sputter guns in a side-sputter configuration. This second system will extend our deposition capabilities.

\section{Investigators' Time and budget}

The investigators spent the expected time on the contract, with a slight modification. V.L. Highland worked on the project full time for two summer months and had une-third released time durin", the academic year. L.B. Auerbach's invoiviment was slightly reduced: one-third time during the academic year, and one summer month. K. McFarlane had a leave for the academic year 89-90 and devoted full time to the project from July 1 st 1989 till June 30th 1990, with half salary from Temple 


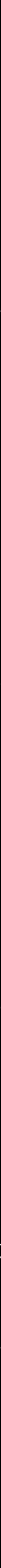


and half from DOE. Beginning July 1st 1990, he will work at the SSC Laboratory, but will devote $25 \%$ of his time to this project. Salary will come $40 \%$ from Temple, $60 \%$ from the SSC.

In Septemher 1988, C.J. Martoff joined the Physics Department at Temple as a Visiting Associate Professor. $\mathrm{H} r$ has worked (about one-sixth time during the academic year and one month in the summer) on the LCD and CEBAF parts of this project, while preparing an independent proposal. He has now been appointed to a tenure-track position and has submitted independent proposals.

We have been fortunate in attracting excellent Post-Doctoral Research Invesigators. We were joined at the beginning of thecurrent period by S. Kettell (Ph.D. from Yale on muonium work at Los Alamos) and C. Guss (Ph.D. from Northwestern on $\sigma_{\text {tot }}$ for $\mathrm{p} \overline{\mathrm{p}}$ at $1.8 \mathrm{TeV}$, at Fermilab).

G. Daniel has played an important role as Senior Research Technician; he is University supported. Students working on the project included John Belz, on $K_{L}^{0} \rightarrow \mu e$, and Y. Zhang, T. Garzarella, C. Fuller, X. Wang and D. Works on the superconducting detector development.

Domestic and foreign travel was close to what was planned.

A detailed financial report will be filed at the appropriate time.

\section{Publications and Other References}

\section{References}

(1) New Experimental Limits on $K_{r}^{0} \rightarrow \mu e$ and $K_{L}^{0} \rightarrow$ ee Branching Ratios, C. Mathiazhagan, W.R. Molzon, R. D. Cousins, J. Konigsberg, J. Kubic, P. Melese, P. Rubin, W. E. Slater, D. Wagner, G. W. Hart, W. W. Kinnison, D. M. Lee, R. J. McKee, E. C. Milner, G. H. Sanders, H. J. Ziock, K. A risaka, P. Knibbe, J. Urheim, S. Axelrod, K. A. Biery, G. M. Irwin, K. Lang, J. Margulies, D. A. Ouimette, J. L. Ritchie, Q. H. Trang, S. G. Wojcicki, L. B. Auerbach, P. Buchholz, V. L. Highland, W. K. McFarlane, M. Sivertz, M. D. Chapman, M. Eckhause, J. F. Ginkel, A. D. Hancock, D. Joyce, J. R. Kane, C. J. Kenney, W. F. Vulcan, R. E. Welsh, R. J. Whyley, R. G. Winter, Temple Report DOE-ER:0389-59, Physical Review Letters 83, 2181 (1989)

(2) Measurement of the Branching Ratio for the Decay $K_{L}^{0} \rightarrow \mu \mu$, C. Mathiazhagan, W.R. Molzon; R. D. Cousins, J. Konigsberg, J. Kubic, P. Melese, P. 
Rubin, W. E. Slater, D. Wagner, G. W. Hart, W. W. Kinnison, D. M. Lee, R. J. McKee, E. C. Milner, G. H. Sanders, H. J. Ziock, K. Arisaka, P. Knibbe, J. Urheim, S. Axelrod, K. A. Biery, G. M. Irwin, K. Lang, J. Margulies, D. A. Ouimette, J. L. Ritchie, Q. H. Trang, S. G. Wojcicki, L. B. Auerbach, P. Buchholz, V. L. Highland, W. K. McFarlane, M. Sivertz, M. D. Chapman, M. Eckhause, J. F. Ginkel, A. D. Hancock, D. Joyce, J. R. Kane, C. J. Kenney, W. F. Vulcar, R. E. Wielsh, R. J. Whyley, R. G. Winter, Temple Report DOE-ER40389-60, Physical Review Letters 63, 2185 (1989)

(3) The Response of an Aluminum-Scintillator Calorimeter to Electrons near the Critical Energy from 50 to $150 \mathrm{MeV}$ and Comparisons with the EGS4 program predictions, L. Auerbach, S. Clearwater, T. Dombeck, T.J. Bowles, H.S. Mattis, W. Gorn, B. Dieterle, J. Kang, C. Leavitt, and D. Koetke, LANL report LA-UR-89-2978 and Temple Report DOE-ER40389-55. Nuclear Instruments and Methods in Physics Research A287, 378 (1990)

(4) Status of BNL E791: Study of very rare $K_{L}^{0}$ decays, W.K. McFarlane, Twelfth International Workshop on Weak Interactions and Neutrinos, April 9-14, 1989, Ginosar, Sea of Galilee, Israel. Temple Report DOE-ER40389-54 and Nuclear Physics B (Proc. Suppl.) 13, 571 (1990).

[5] P. Zecher, D. Wang, J. Rapaport, C.J. Martoff, B.A. Young, Phys Rev A 41, 4058 (1990).

\subsection{Talks and Conference Reports}

[6] "Radioactive Contamination of Pure Materials", C.J. Martoff, Invited talk delivered at CFPA Workshop on Ultra Low Background Experiments, Center for Particle Astrophysics, University of California, Berkeley, December, 1989.

17) "Dark Matter and Low Energy Particle Physics", C.J. Martoff, Physics and Astronomy Department Colloquium, Michigan State University, East Lansing, MI January 1990.

|8| "Cryogenic Thin Film Particle Detecucrs", C.J. Martoff, Invited talk at Rutgers University Physics Department meeting on DIAMAS- A Compact Diamond-Based Detector for the SSC, Piscataway, N.J. , May 1990 (unpublished). 


\subsection{Other References}

[9] E791 collaboration:

C. Mathiazhagan, W.R. Molzon

University of California, Irvine, California 92717

K. Arisaka, R. D. Cousins, T. Kaarsberg, J. Konigsberg, J. Kubic, P. Rubin, W. E. Slater, D. Wagner

University of California, Los Angeles, California 90024

G. W. Hart, W. W. Kinnison, D. M. Lee, R. J. McKee, E. C. Milner, G. H. Sanders, H. J. Ziock

Los Alamos National Laboratory, Los Alamos, New Mexico 87545

P. Knibbe, J. Urheim

University of Pennsylvania, Philadelphia, Pennsylvania 19104

K. A. Biery, G. M. Irwin, K. Lang, J. Margulies, D. A. Ouimette, S. G. Wojcicki Stanford University, Stanford, California 94909

L. B. Auerbach, P. Buchholz, C. Guss, V. L. Highland, S. Kettelll, W. K. McFarlane, M. Sivertz

Temple University, Philadelphia, Pennsyluania 19122

J. L. Ritchie, G. Hoffman, P. Riley

University of Texas at Austin, Austin, TX 78712

M. D. Chapman, M. Eckhause, J. F. Ginkel, A. D. Hancock, J. R. Kane, C. J. Kenney, W. F. Vulcan, R. E. Welsh, R. G. Winter

College of William and Mary, Williamsburg, Virginia 29185

(10) Cousins, R.D., P. Melese, (UCLA); J.F. Frank, W. Kinnison, J.W. Lillberg, R. McKee Jr., G. Sanders, (LANL); W.R. Molzon, W.D. Wales (U of Pennsylvania); J.F. Greenhalgh, (Princeton U); G.M. Irwin, J.L. Ritchie, S.G. Wojcicki (Stanford U); L.B. Auerbai'h, V.L. Highland, W.K. McFarlane (Temple University). Added after subrission: J. Konigsberg, W. Slater, P. Rubin, (UCLA); D.M. Lee, C. Milner, H. Ziock (LANL); K. Arisaka, J. Urheim, P. Knibbe (U of Pennsylvania); K. Lang, S. Axelrod, J. Margulies, (Stanford); P. Buchholz, M. Sivertz (Temple U); and R. Welsh, M. Eckhause, R. Winter, C. Kenney, M. Chapman, J. Ginkel, R. Whyley, P. Guss, D. Joyce, J.R. Kane (College of Wrn. and Mary), Proposal to AGS: Study of Very Rare K Decays. Submitted January 1984, approved by BNL as E791, June 1984.

(11) The LCD (LAMPF E1015) project is described in: 
"A proposal for a precision test of the Standard Model by neutrino-electron scattering," R.C. Allen et al., UC Irvine, UCLA, UC Riverside, U of Colorado, CEBAF, T.ANL, UNM, U of Penn, Temple U, and Wm. \& Mary. LA-11300-P, UC-410 and Temple report DOE-ER40389-52 (April 1988).

[12] A proposal to Search for Neutrino Oscillations with High Sensitivity in the Appearnce Channels $\nu_{\mu} \rightarrow \nu_{e}$ and $\bar{\nu}_{\mu} \rightarrow \bar{\nu}_{e}$, X.-Q. Lu et al., UC Irvine, UC Riverside, CEBAF, Los Alamos Nat. Lab., Louisiana State U, UNM, U. of Penn., and Temple U. June 30th 1989 and Dec. 1st 1989. LAMPF proposal E1173

[13] Measurement of Parity Violation in Elastic polarized-ep Scattering, R. Carlini et al., PAVEX collaboration (CEBAF, Hampton U., Harvard, Illinois, LANL, Mississippi, MIT, Syracuse, Temple, and Wm. \& Mary), Proposal PR-89-023 to CEBAF, October 31st, 1989.

[14] Development of High-Yield Process for Fabrication of Low-T Trilayer Tunnel Junctions by Multitarget Sputtering and Reactive Ion Etching, Kurt J. Lesker Company, 1515 Worthington Ave., Clairton, PA 15025 with Prof. C.J. Martoff, proposal to the Ben Franklin Partnership Fund, Small Business Research Grant Program, of the Commonwealth of Pennsylvania, Sept. 89 , requesting $\$ 34,878$ and committing $\$ 189,428$ in company funds.

(15) Search for the Decays $K_{L}^{0} \rightarrow \mu e$ and $K_{L}^{0} \rightarrow e e$, R.D. Cousins, J. Konigsberg, J. Kubric, P. Melese, P.Rubin, W.E. Slater, J.S. Frank, G.W. Hart, W.W. Kinnison, D.M. Lee, E.C. Milner, G.H. Sanders, H.J. Ziock, K. Arisaka, P. Knibbe, W.R. Molzon, J. Urheim, W.D. Wales, S. Axelrod, K.A. Biery, G. Bonneaud, G.M. Irwin, K. Lang, C.J. Martoff, D.A. Ouimette, J.L. Ritchie, Q.H. Trang, S.G. Wojcicki, L.B. Auerbach, P. Buchholz, V.L. Highland, W.K. McFarlane, M.B. Sivertz, M.D. Chapman, M. Eckhause, J.F. Ginkel, P.P. Guss, D. Joyce, J.R. Kane, C.J. Kenney, W.F. Vulcan, R.E. Welsh, R.J. Whyley, and R.G. Winter, Phys. Rev. D38, 2914 (1988)

(16) Facility for Development of Superconducting Detectors for Nuclear Radiation, C.J. Miáitoff, J. Crow and K.W. McFarlane, DOE proposal and Temple report DOE-ER $40389-63$

[17| E. Jastrzembski et al., Physical Review Letters 61, 2300 (1988). 
[18] T. Inagaki, M. Kobayashi, T. Sato..., Physical Review D40, 1712 (1989).

[19] R.N. Cahn and H. Harari, Nucl. Fìys. B176, 135 (1980).

(20) Buchmuller, W., and D. Wyler, Nucl. Phys., B268, 621 (1986).

[21] M. Zeller, XXIV Int'l. Conf. on H.E. Physics, Munich, Aug. 1988.

[22] U. Bellgardt et al., Nucl. Phys. B299, 1 (1988).

[23] D.A. Bryman et al., Phys. Rev. Lett. 55, 465 (1985)

[24] R.D. Bolton et al., Phys. Rev. Lett. 56, 2461 (1986)

(25) L. M. Sehgal, Phys. Rev. 183, 1511 (1969); B. R. Martin, E. de Rafael, and J. Smith, Phys. Rev. D2, 179 (1970). Earlier work on the analogous decays of the $\pi^{0}$ included S. M. Berman and D. A. Geffen, Nuovo Cimento 18, 1192 (1960); D. A. Geffen and B. L. Young, Phys. Rev. Lett. 15, 316 (1965).

[26] W.C. Carithers et al., Phys. Rev. Lett. 30, 1336 (1973); W.C. Carithers et al., Phys. Rev. Lett. 31, 1025 (1973); Y. Fukushima et al., Phys. Rev. Lett. 36, 348 (1976); M.J. Shochet et al., Phys. Rev D 19, 1965 (1979).

[27] S. Ahmad et al., Phys. Rev. Lett. 59, 970 (1987).

[28] R. E. Shrock and M. B. Voloshin, Phys. Lett. 87B, 375 (1979); T. Inami and C. S. Lim, Prog. Theor. Phys. 65, 297 (1981); N. F. Nasrallah and K. Schilcher; Z. Phys. C36, 467 (1987).

[29] J.D. Walecka, "Semileptonic weak interactions in nuclei," in Muon Physics, Vol. II, p.350, eds. V.W. Hughes and C.S. Wu, Academic Press (NY, 1975).

[30 M.W. Goodman and E. Witten, Phys Rev D31, 3059 (1985)

(31) D.Z. Freedman et al, Ann Rev.Nuc Sci 27, 167 (1977) 

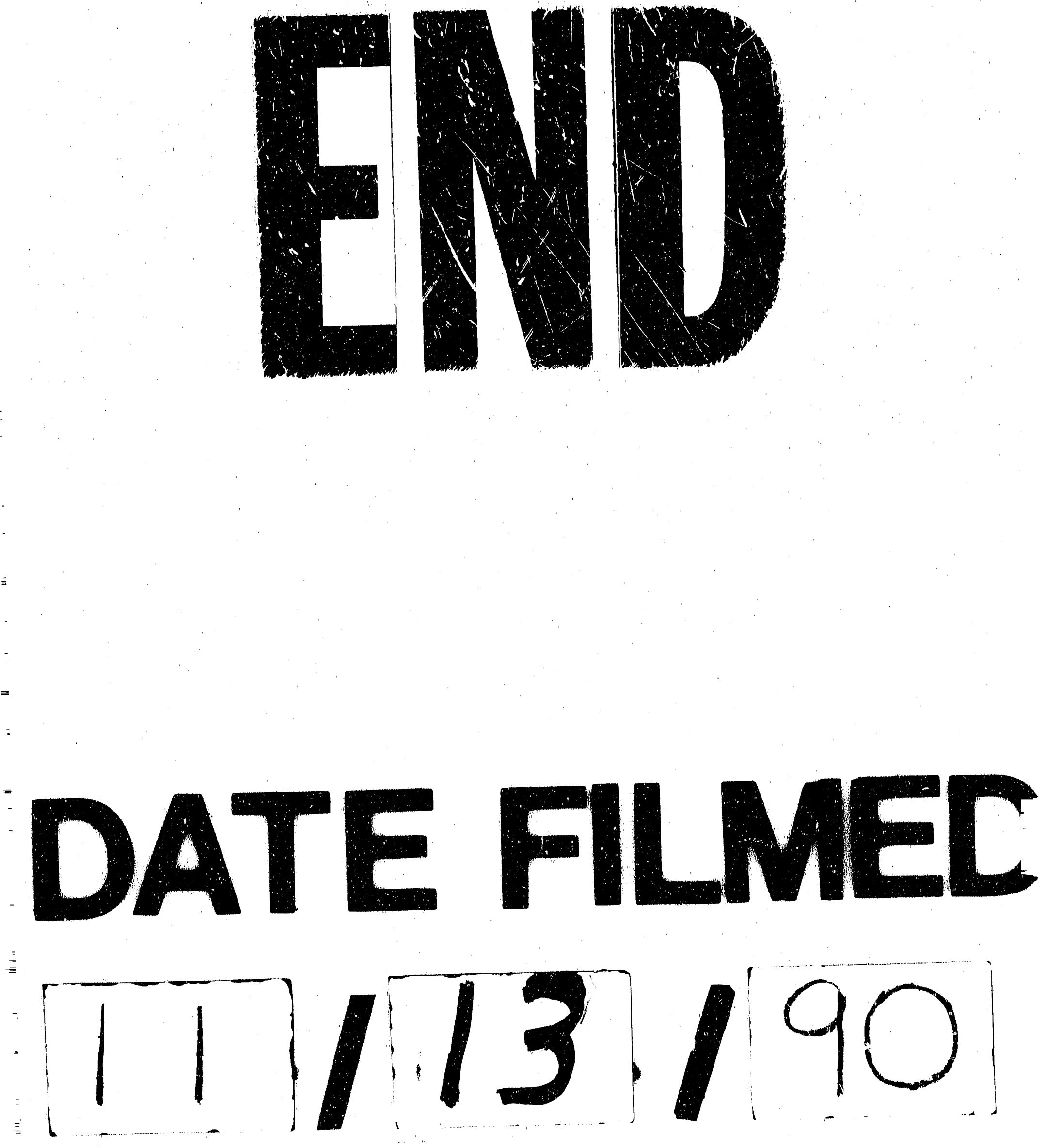
